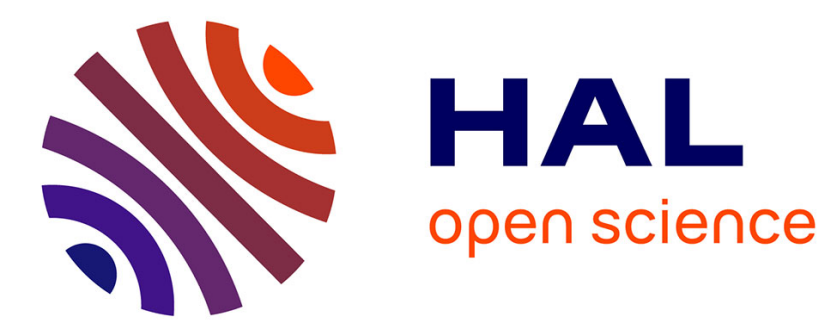

\title{
Covering a 3D flat surface with autonomous and mobile wireless sensor nodes
}

Nadia Boufares, Pascale Minet, Ines Khoufi †, Leila Saidane

\section{To cite this version:}

Nadia Boufares, Pascale Minet, Ines Khoufi $\dagger$, Leila Saidane. Covering a 3D flat surface with autonomous and mobile wireless sensor nodes. IWCMC 2017 - the 13th International Wireless Communications and Mobile Computing Conference, Jun 2017, Valencia, Spain. hal-01640508

\section{HAL Id: hal-01640508 \\ https://hal.science/hal-01640508}

Submitted on 20 Nov 2017

HAL is a multi-disciplinary open access archive for the deposit and dissemination of scientific research documents, whether they are published or not. The documents may come from teaching and research institutions in France or abroad, or from public or private research centers.
L'archive ouverte pluridisciplinaire HAL, est destinée au dépôt et à la diffusion de documents scientifiques de niveau recherche, publiés ou non, émanant des établissements d'enseignement et de recherche français ou étrangers, des laboratoires publics ou privés. 


\title{
Covering a 3D flat surface with autonomous and mobile wireless sensor nodes
}

\author{
Nadia BOUFARES*, Pascale MINET ${ }^{\dagger}$, Ines KHOUFI ${ }^{\dagger}$, Leila SAIDANE* \\ *National School for Computer Science, Manouba, Tunisia. \\ $\dagger$ Inria, 2 rue Simone Iff, CS 42112, 75589 Paris Cedex 12, France. \\ nedya.boufares@ensi-uma.tn, pascale.minet@inria.fr, ines.khoufi@inria.fr, leila.saidane@ensi-uma.tn,
}

\begin{abstract}
Wireless Sensor Networks (WSNs) are used in a wide range of applications due to their monitoring and tracking abilities. Depending on the applications goals, sensor nodes are deployed either in a two dimensional (2D) area or in a threedimensional (3D) area. In addition, WSN deployment can be either in a distributed or a centralized manner. In this paper, we are interested in a fully distributed deployment of WSN in several 3D-flat-surface configurations using autonomous and mobile nodes. Our goal is to ensure full 3D flat surfaces coverage and maintain network connectivity for these surfaces. To reach our goal we propose 3D-DVFA-FSC, a distributed deployment algorithm based on virtual forces strategy to move sensor nodes over different 3D-flat-surface shapes. Simulation results show that 3D-DVFA-FSC provides a full coverage rate regardless of the 3D-flat-surface configuration while maintaining network connectivity.
\end{abstract}

\section{Context And Motivation}

Recent advanced researches on industrial and commercial applications have enabled the emergence of a new cutting-edge network of small and low-power devices which is the Wireless Sensor Network (WSN). Sensor nodes are scattered in the field of interest in order to monitor environmental and physical phenomena such as temperature, pressure, vibrations... and to track moving targets such as enemy vehicles, intruders, animals...Hence, WSNs are solicited in several applications including underwater acoustic monitoring, weather and environment disaster forecasting, military battlefields, home automation... To do so, node deployment is considered as the main issue in WSNs, since it affects the lifetime and Quality of Service (QoS) for WSNs. To provide an optimized deployment, two key requirements should be taken into consideration: the area coverage and network connectivity. The area to be supervised is said fully covered if each event occurring in this area will be detected by at least one sensor node. If there exists at least one path between each node and a central node collect sink then the network connectivity is said maintained so that any encountered event will be reported to the sink. The requirements that must be met by a network deployment depend mostly on the application supported. Some applications need a full coverage and a permanent connectivity such as in military battlefields. For other applications, partial coverage and temporary connectivity are enough. In order to meet all these requirements, a deployment algorithm is needed to determine the appropriate sensor node positions. The deployment algorithm can be centralized, where the sink node is the entity that collects different nodes positions and then computes the new positions of nodes in the new deployment. Later, a human or a mobile robot will be in charge of moving nodes to their new positions. Otherwise, if sensor nodes are mobile and autonmous, then, the algorithm is distributed and nodes will collaborate and exchange data to determine their new positions and achieve a new deployment. Extensive previous researches on coverage and connectvity issues focused mainly on terrestrial applications but recently it seems that the elevation of nodes plays a very significant role in the WSN deployment. Most of these works took into consideration a 3D space where nodes are located freely within the whole field of interest as shown in Figure 1a. However, in many real world applications, this area is neither horizontal flat 2D nor 3D space. Instead, it is a sequence (continuation) of sloping surfaces (i.e, inclined planes) as depicted in Figure $1 \mathrm{~b}$ or complex 3D surface as shown in Figure 1c. Volcanos monitoring (e.g mountains) and snow tracking are the most relevant 3D surface covering applications.

In this paper, we are interested in scattering mobile nodes over a 3D flat surface. We started by fixing the parameters of the deployment algorithm considering a horizontal 2D plane. Then, we extend the results to cope with 3D inclined plane. We study several shapes of 3D-flat-surfaces. Nodes must move to cover the surface of the given shape. That is why we propose to design a $3 \mathrm{D}$ distributed deployment algorithm that aims to deploy sensor nodes over the 3D surface area in order to ensure full are coverage and network connectivity. This algorithm is based on the virtual forces approach [9]. This paper is organized as follows: In Section II, we give an overview of the state of the art. Section III describes our 3D distributed virtual forces algorithm in details. Section IV deals with the performance evaluation of our algorithm. Finally, we conclude and discuss future work in Section V.

\section{RELATED WORK}

In the literature, several strategies are proposed to determine optimal nodes positions in order to provide high coverage rate. Extensive studies were interested in terrestrial deployments. These strategies focused mainly on 2D applications.

Recent studies [1] showed that 3D surface deployment is completely different from deployments in a $2 \mathrm{D}$ plane or a $3 \mathrm{D}$ 


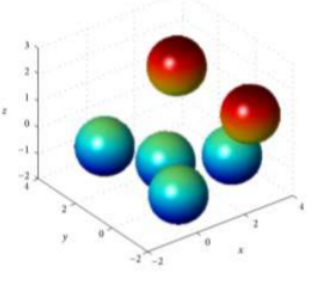

a $.3 \mathrm{D}$ volume

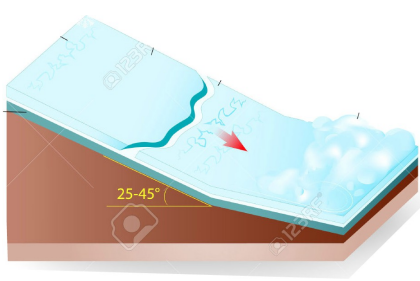

b. $.3 \mathrm{D}$ flat surface

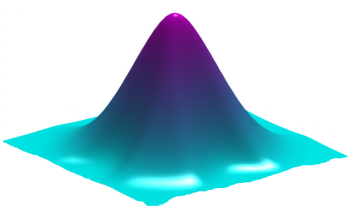

c .3D complex surface

Fig. 1: 3D area

volume. Existing 2D and 3D solutions could not be directly extended to 3D surface covering schemes otherwise coverage holes will arise. Figure 2 shows that if the deployment of the 2D sensor nodes on the horizontal plane depicted in blue is used to build by projection in the deployment on the sloping plane depicted in red, coverage holes appear in both cases of projection.

If $d$ the distance between two blue neighboring nodes is increased by projection $\left(d^{\prime}>d\right.$ on Figure 2a), these nodes projected in the red plane are no longer neighbors.

If on the contrary, the distance is decreaed (see Figure 2b), coverage holes occur in the upper part of the red plane.

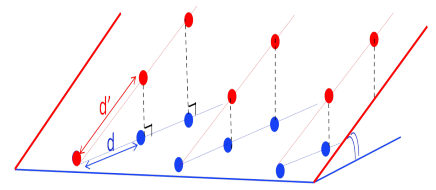

a Planar coverage holes case 1

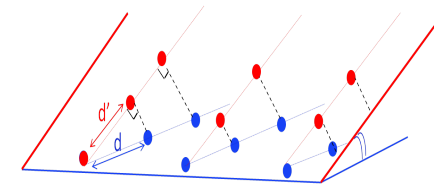

b Planar coverage holes case 2
Fig. 2: Planar coverage holes

Hence, 3D surfaces, whether 3D flat surfaces or 3D complex surfaces, come out with new challenges such as how to handle variations in the shape of the 3D surface.

There are different ways to classify existing research on the coverage-connectivity problem. We distinguish two main deployment strategies: static strategies and dynamic strategies. In static approaches, we can adopt the grid-based strategy [2]. With the grid-based strategy, if the area is considered regular, then, nodes must be placed either at cell centers or at cell vertices. However, if the region has irregular borders, in order to avoid coverage holes, extra positions are added out of the grid. Many researches were held to determine the best space-filling polyhedron that tessellates a 3D space with the highest volumetric quotient. In fact, a polyhedron is a three-dimensional shape that consists of a finite number of polygonal faces such as the triangular prism, the hexagonal prism, the cube, the truncated octahedron... The authors in
[3] showed that the trucated octahedron tessellation is the best tessellation for ensuring a $3 \mathrm{D}$ volume coverage using the minimum number of sensor nodes. Nodes will be placed at the centers of trunacted octahedron cells. They demonstrated, then, that the communication range $\mathrm{R}$ must be at least 1.7889 times the sensing range $r$ to maintain the network connectivity.

On the other hand, many studies focused on dynamic deployments where nodes are considered as mobile nodes. Authors, in [5], used Voronoi cells to ensure 2D or 3D kcoverage (i.e. an area is said k-covered if each each unit of the space considered is covered by at least $\mathrm{k}$ sensor nodes). They illustrated APOLLO deployment, an autonomous deployment to provide $\mathrm{k}$-surface coverage. With this approach, nodes are able to move with the intention of achieving k-coverage while minimizing the maximum sensing range required by the nodes. If the computational geometry strategy is adopted, then, nodes positions are computed using the Voronoi diagram or the Delaunay triangulation. This approach has a high cost of computation. The authors in [4] tried to find the optimal deployment on different 3D surfaces that will achieve a high sensing quality. They proved that the Centroidal Voronoi tessellation leads to the required optimal deployment. However, this approach is not scalable since nodes positions are computed by the sink.

The virtual forces paradigm is a distributed algorithm which is based on attractive and repulsive forces. Sensor nodes move to their new positions according to a predefined threshold distance $D_{t h}$ that should be maintained between neighbors during the network lifetimne. If the distance between two sensor nodes is less than $D_{t h}$, then, the neighbor node will exert a repulsive node. If this distance is greater than $D_{t h}$, an attractive force is exerted. Otherwise, the force is null. Each node computes its resultant force autonomously and then, determines its new position depending on that force. 2D virtual forces algorithm has been extended to the 3D version and used in both [6], [7] and [8], aiming to ensure 3D space full coverage of 3D area and maintain network connectivity. The virtue of this approach consists in its simplicity: sensor nodes are able to spread and move in the whole area so that they can meet rapidly the coverage and connectivity requirements. In this paper, we focus on 3D flat surface coverage. We assume that the 3D surface may be unknown or hostile and sensor nodes are mobile and autonomous. We adopt the virtual forces strategy to allow nodes spreading over the whole 3D surface (e.g. mountains). The goal is to ensure full 3D surface coverage and maintain network connectivity.

\section{Our Contribution : 3D-DVFA-FSC}

To deploy sensor nodes in a 3D-surface, we propose 3DDVFA-FSC algorithm: 3D Distributed Virtual Forces Algorithm for Flat Surface Covering. 3D-DVFA-FSC is a distributed algorithm based on the virtual forces strategy to allow nodes spreading on the 3D-surface to achieve full coverage and connectivity. The main goal of the virtual forces paradigm is to 
maintain the same target distance between neighboring nodes. This target distance is called $D_{t h}$, the Distance threshold.

\section{A. Models and Assumptions}

In this paper, we adopt the following assumptions in order to cover the whole 3D surface:

- Each node in the sensor network can determine its own position from global positioning system (GPS).

- The binary sensing model will be adopted as the sensing model, which means the probability of detecting the target is 1 if this one is within the sensor's sensing range, and 0 otherwise.

- The communication model is also binary.

- The sensing range and communication range are modeled by two spheres of radius $r$ and $R$ respectively.

- The entity to monitor is a sequence of sloping surfaces.

\section{B. Principles of $3 D-D V F A-F S C$}

The 3D-DVFA-FSC algorithm proceeds in iterations. At each iteration, each node broadcasts a Hello message. In the Hello message, each node sends its position and listens to incoming Hello messages in order to perform the neighborhood discovery. Then, each sensor node $s_{i}$ is able to determine its 1-hop neighbors and 2-hop neighbors. These neighbors will exert either attractive or repulsive force, according to their distance to node $s_{i}$ that will be able to compute its new position depending on the resultant force.

Let $d_{i j}$ denote the euclidean distance between the sensor nodes $s_{i}$ and $s_{j}$.

$d_{i j}$ is given by $\sqrt{\left(x_{j}-x_{i}\right)^{2}+\left(y_{j}-y_{i}\right)^{2}}$.

The force exerted by sensor $s_{j}$ on sensor $s_{i}$ is

- an attractive force if $d_{i j}>D_{t h}$ and is given by:

$$
F_{i j}=K_{a} \cdot\left(d_{i j}-D_{t h}\right) \cdot \frac{\left(x_{j}-x_{i}, y_{j}-y_{i}\right)}{d_{i j}}
$$

- a repulsive force, if $d_{i j}<D_{t h}$ and is equal to

$$
F_{i j}=K_{r} \cdot\left(D_{t h}-d_{i j}\right) \cdot \frac{\left(x_{i}-x_{j}, y_{i}-y_{j}\right)}{d_{i j}}
$$

- null otherwise, $\left(d_{i j}=D_{t h}\right)$

Hence, the resultant force $F_{i}$ on $s_{i}$ is computed as the sum of these forces exerted by its 1-hop and 2-hop neighbors:

$$
F_{i}=\sum_{j} F_{i j}
$$

The sensor nodes should move on the given 3D surface depending on the resultant force. Notice that $K_{a}$ and $K_{r}$ are the attractive coefficient and the repulsive coefficient of the virtual forces strategy, respectively. To favor the convergence of the algorithm, $K_{r}$ should be much higher than $K_{a}$. To improve the efficiency of the algorithm, the distance traveled by each sensor node at each iteration is limited by Dist $_{\text {max }}$, a parameter of the 3D-DVFA-FSC algorithm.
Initially, nodes are scattered at the bottom of the 3D flat surface, except for the fourth configuration 4, where nodes are scattered at the top of the surface.

As the resultant force is computed as a $2 \mathrm{D}$ planar vector, then to determine accurately the new position onto the 3D sloping surface, sensor nodes should travel on the three dimensional surface the same distance that would be traveled in 2D horizontal surface (without taking into account the height) as illustrated in Figure 3. In case of continous 3D flat surfaces as illustrated in configuration 3 and configuration 4 , sensor nodes move to an intermediate position which represents the edge between the two flat areas then moves towards the next flat area. The sum of the traveled distances should not exceed the computed 2D distance (without taking into account the height) as depicted in Figure 4.

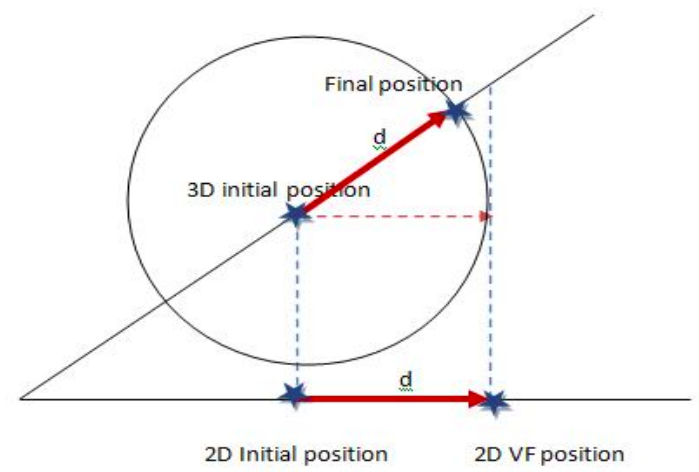

Fig. 3: 3D-flat-surface Virtual force iteration

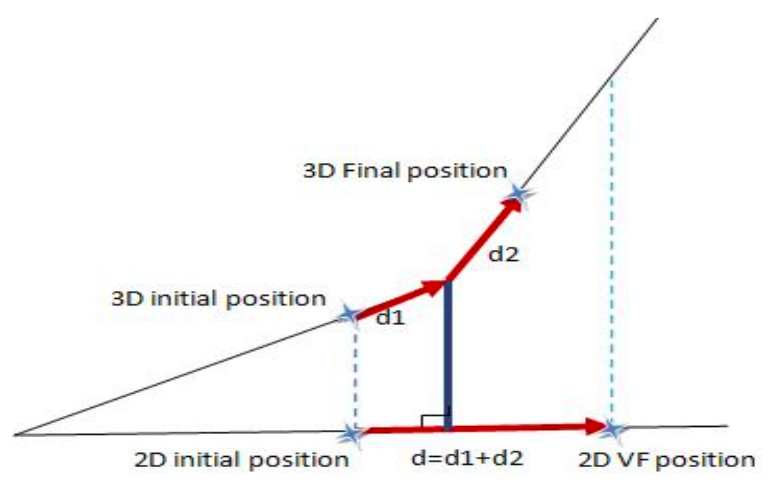

Fig. 4: 3D-continous-surface Virtual force iteration

The distributed algorithm proceeds as follows. Each sensor node computes the resultant of the virtual forces according to the principle given previously, taking into account only the dimensions $\mathrm{x}$ and $\mathrm{y}$. Then it moves according to the direction of the resultant force while following the shape of the surface.

\section{Simulation Parameters}

In real applications, when dealing with 3D surfaces (e.g, mountains), nodes are scattered randomly, by an aircraft, for instance. Hence, we evaluate 3D-DVFA-FSC using an initial 
configuration where nodes are placed randomly at the bottom of the 3D flat surface.

To evaluate performances of our 3D-DVFA-FSC algorithm, we implemented it in the Network Simulator NS3 version 3.25. Table I illustrates the simulation parameters used to evaluate the 3D-DVFA-FSC algorithm. The values of $K_{a}$ and $K_{r}$ are tuned by series of simulations. The value of $D_{t h}$ is computed according to the triangular lattice deployment. $D_{t h}$ is given by Equations 1 and 2 where the value of the sensing range is equal to $25 \mathrm{~m}$ and the value of the communication range is equal to $50 \mathrm{~m}$.

$$
\begin{aligned}
& R c>=\sqrt{3} * R s \\
& R s=\sqrt{3} * D_{t h}
\end{aligned}
$$

TABLE I: Simulation parameters

\begin{tabular}{cc}
\hline \hline Topology & \\
\hline \hline Number of nodes & 250 \\
Speed & $5 \mathrm{~m} / \mathrm{s}$ \\
\hline \hline MAC Layer & \\
\hline \hline Protocol & IEEE $802.11 \mathrm{~b}$ \\
Throughput & $2 \mathrm{Mb} / \mathrm{s}$ \\
Transmission range $R$ & $50 \mathrm{~m}$ \\
Sensing range $r$ & $25 \mathrm{~m}$ \\
\hline \hline Simulation & \\
\hline \hline Result & average of 20 simulation runs \\
Simulation time & $3000 \mathrm{~s}$ \\
\hline \hline 3D-DVFA & \\
\hline \hline Ka & 0.007 \\
Kr & 0.3 \\
Hello period & $2 \mathrm{~s}$ \\
Dth & $43.3 \mathrm{~m}$ \\
Distmax & $D t h / 6$ \\
\hline \hline
\end{tabular}

To evaluate the effeciency of our solution, we studied different shapes of flat surfaces inclined at various angles and having an area of $250000 \mathrm{~m}^{2}$. The height of the surface will be calculated according to the planar equation $z=x * \tan (\alpha)$, where $\alpha$ denotes the angle of the surface with the horizontal plane. Nodes are scattered initially at the bottom of the mountain.

In the first configuration, the $3 \mathrm{D}$ flat surface considered is depicted in Figure 5a. Its equation is given by: $z=x * \tan (\alpha 1)$, with $\alpha 1=30^{\circ}$.

In the second configuration, the 3D flat surface considered is depicted in Figure 5b. Its equation is given by: $z=x * \tan (\alpha 2)$, with $\alpha 2=60^{\circ}$.

In the third configuration, the flat 3D surface considered is depicted in Figure 6a. Its equation is given by:

$$
\begin{array}{lrr}
z=x * \tan (\alpha 1) & \text { if }(x>=0 & x<=216.5) \\
z=(x * \tan (\alpha 2))-250 & \text { if }(x>=216.5 & x<=341.5)
\end{array}
$$

In the fourth configuration, the flat $3 \mathrm{D}$ surface considered is depicted in Figure 6b. Its equation is given by:

$$
\begin{array}{lrr}
z=(-x * \tan (\alpha 2))+341.5 & \text { if }(x>=0 & x<=125)) \\
z=(-x * \tan (\alpha 1))+197.16 & \text { if }(x>=125 & x<=341.5)
\end{array}
$$

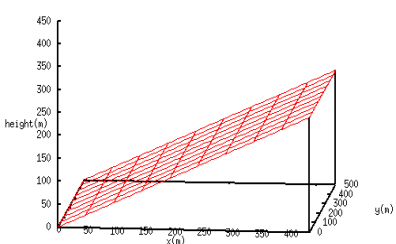

a configuration 1

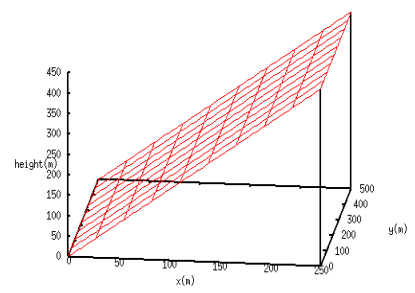

b configuration 2
Fig. 5: 3D flat surfaces

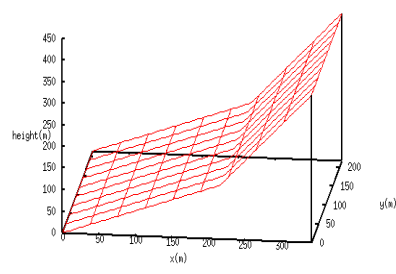

a configuration 3

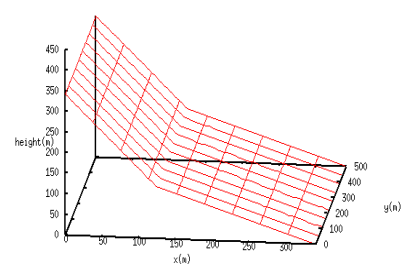

b configuration 4
Fig. 6: 3D continuous flat surfaces

\section{Simulation Results}

1) Coverage rate: We evaluate the coverage rate as a function of time. To do so, we considered a virtual 2D grid of unit cells on the 3D surface. If there exists at least one node whose distance to the cell center is less than or equal to the sensing range, then, the cell is covered. The coverage rate is equal to the number of covered cells over the total number of cells. Figures 7 and 8 show the coverage rate obtained as an average of 20 simulations. Full coverage (i.e 100\% coverage rate) is reached for all the given configurations as shown in the following figures.

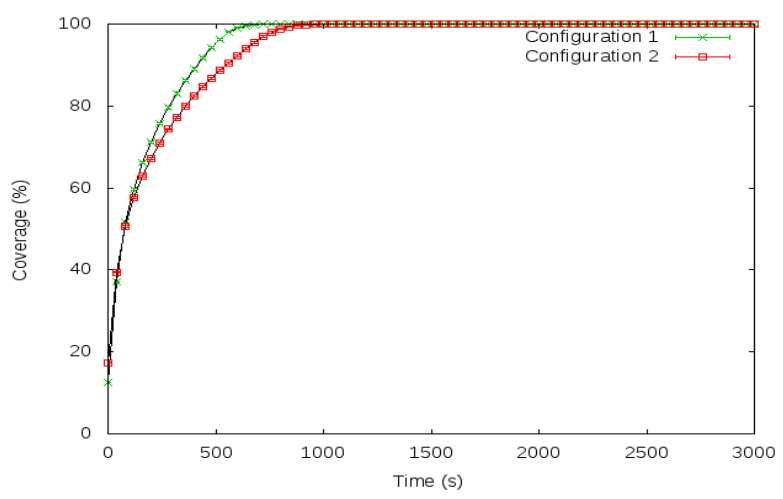

Fig. 7: Coverage rate for configuration 1 and 2 


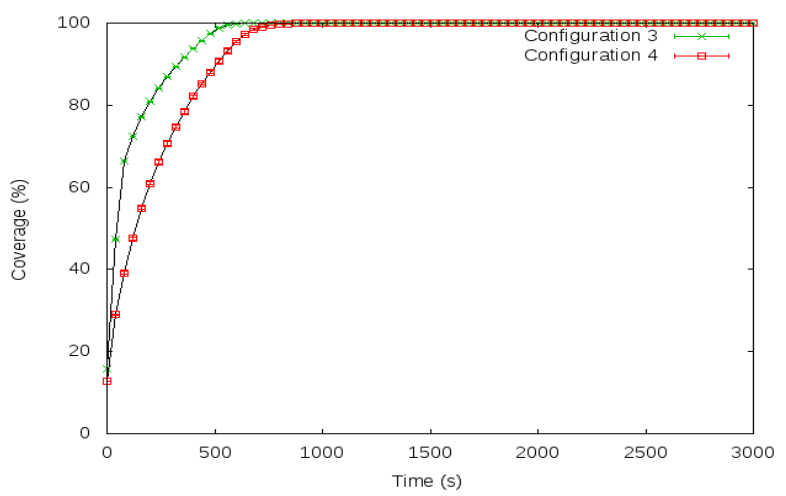

Fig. 8: Coverage rate for configuration 3 and 4

Simulations showed that the virtual forces paradigm is still adopted for 3D flat surface coverage as it was for 2D plane area and for 3D volume if we adapt its parameters $\left(K_{r}, K_{a}, D_{t h} \ldots\right)$. The 3D-DVFA-FSC algorithm enables sensor nodes to heal coverage holes during the deployment iterations. Figures 9 to 12 show both initial and final deployments for the different studied configurations. These figures show that connectivity is achieved at the end of simulation for the four given 3D flat surfaces.

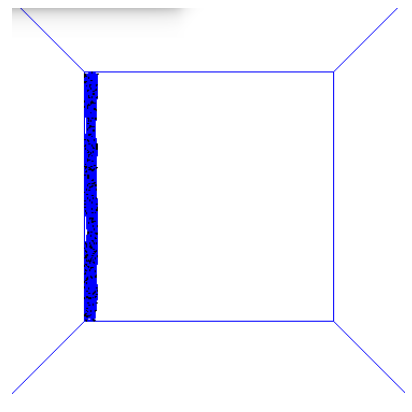

a Initial deployment

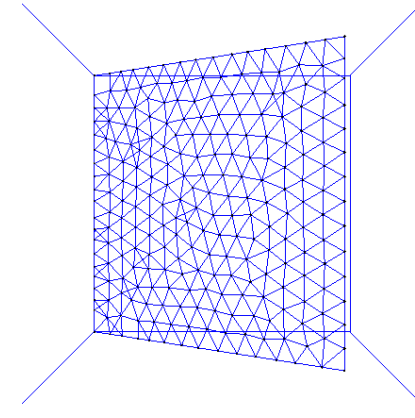

b Final deployment
Fig. 9: First configuration connectivity

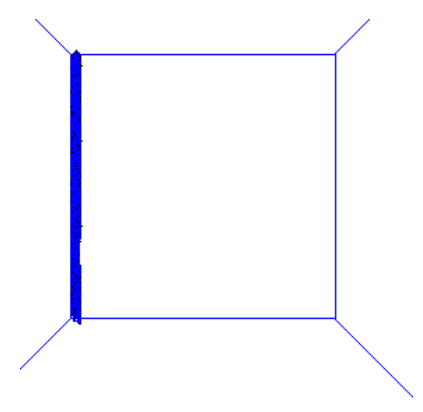

a Initial deployment

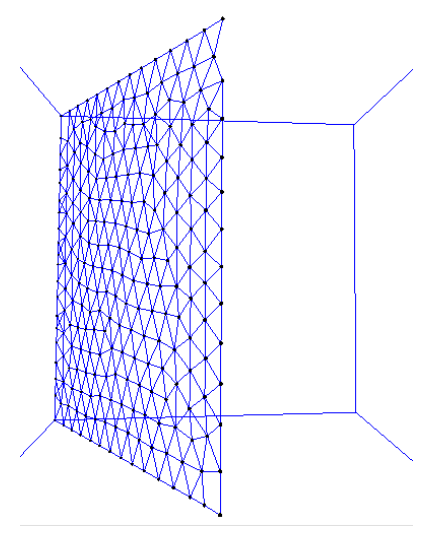

b Final deployment
Fig. 10: Second configuration connectivity

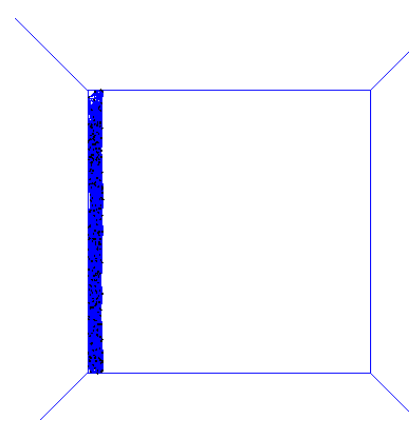

a Initial deployment

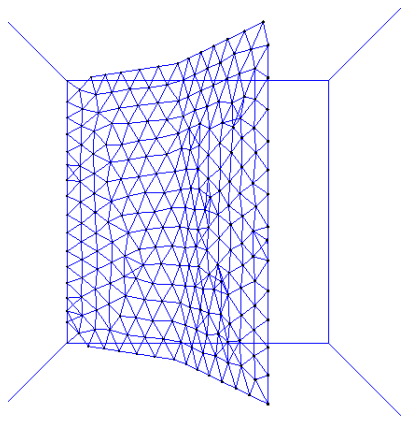

b Final deployment
Fig. 11: Third configuration connectivity

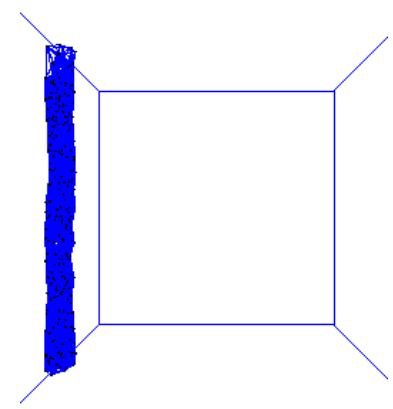

a Initial deployment

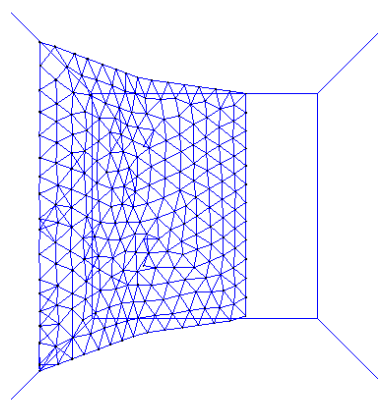

b Final deployment
Fig. 12: Fourth configuration connectivity

\section{E. Distance traveled}

In this paper, we also evaluate the total distance traveled by nodes as a function of time. Figures 13, 14 show the cumulative distance traveled by nodes as a function of time for the different 3D surface scenarios. We can observe that this distance increases as a function of time. This is due to nthe node oscillations problem, the main drawback of the virtual forces strategy. Then, sensor nodes are still oscillating even if the full coverage is ensured. We observe that a higher slope needs more time to ensure coverage but a smalle cumulative traveled distance. For the same number of sensor nodes and the same surface, the deployment seems more regular and optimized with a higher surface slope.

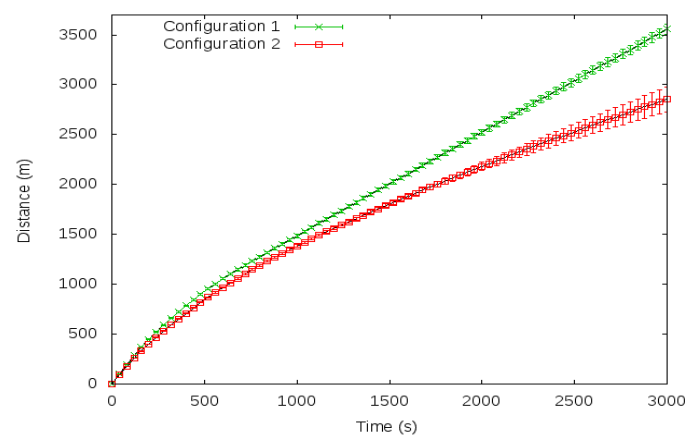

Fig. 13: Distance traveled for configuration 1 and 2 


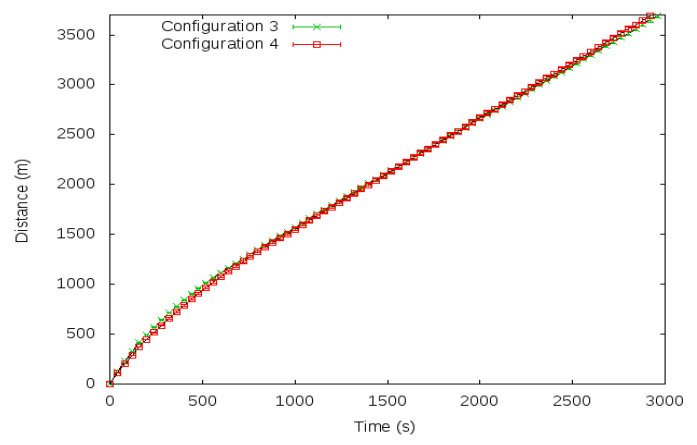

Fig. 14: Distance traveled for configuration 3 and 4

\section{CONCLUSION}

In this paper, we proposed 3D-DVFA-FSC, a distributed deployment algorithm based on virtual forces in order to ensure full 3D-surface coverage and maintain network connectivity. We evaluated 3D-DVFA-FSC using different 3D flat surface shapes. Initially, nodes were randomly deployed. Full coverage was reached in the given configurations and maintain that rate to the end of simulation. We also evaluated the total distance traveled by nodes. Simulation results show that sensor nodes still move even when full 3D-surface coverage is reached. This is due to the node oscillations problem. This problem will be tackled in our future work. We will also focus on how to stop nodes when full coverage is reached and consider 3D surface complex shapes where the challenges of coverage and connectivity are more complicated.

\section{REFERENCES}

[1] M.-C. Zhao, J. Lei, M.-Y. Wu, Y. Liu, and W. Shu, Surface coverage in wireless sensor networks, in IEEE INFOCOM, 2009.

[2] I. Khoufi, P. Minet, A. Laouiti, and S. Mahfoudh, Survey of deployment algorithms in wireless sensor networks: coverage and connectivity issues and challenges, International Journal of Autonomous and Adaptive Communications Systems (IJAACS), p. 24, 2014.

[3] S. Alam and Z. J. Haas, Coverage and connectivity in three- dimensional networks, in Proceedings of the 12th annual international conference on Mobile computing and networking. ACM, pp. 346-357, 2006.

[4] M. Jin, G. Rong, H. Wu, L. Shuai, and X. Guo, Optimal surface deployment problem in wireless sensor networks, in IEEE INFOCOM, pp. 2345-2353, 2012.

[5] F. Li, J. Luo, W. Wang, and Y. He, Autonomous deployment for load balancing-surface coverage in sensor networks, Wireless Communications, IEEE Transactions on, vol. 14, no. 1, pp. 279-293, 2015.

[6] X. Li, L. Ci, M. Yang, C. Tian, and X. Li, Deploying three- dimensional mobile sensor networks based on virtual forces algorithm, in Advances in Wireless Sensor Networks. Springer, pp. 204-216, 2013.

[7] C. Miao, G. Dai, X.-m. Zhao, Z. Tang, and Q. Chen, 3d self- deployment algorithm in mobile wireless sensor networks, in Advances in Wireless Sensor Networks. Springer, pp. 27-41, 2014,

[8] N. Boufares, I. Khoufi, P. Minet, L. Saidane, and Y. Ben Saied, Three dimensional mobile wireless sensor networks redeployment based on virtual forces, in IEEE Wireless Communications and Mobile Computing Conference (IWCMC), pp. 56-568, 2015.

[9] Y. Zou and K. Chakrabarty, Sensor deployment and target localization based on virtual forces, in INFOCOM 2003. Twenty-Second Annual Joint Conference of the IEEE Computer and Communications. IEEE Societies, vol. 2. IEEE, 2003, pp. 1293-1303.

[10] N. Aslam and W. Robertson, "Distributed coverage and connectivity in three dimensional wireless sensor networks," in Proc. ACM IWCMC'10, pp. 1141-1145, 2010. 